\title{
A Bound for the Fixed-Point Index of an Area-Preserving Map with Applications to Mechanics*
}

\author{
Carl P. Simon (Ann Arbor)
}

Area-preserving maps and flows play an essential role in the study of motions of mechanical systems, especially in celestial mechanics (see $[1,14])$. Since one is often interested in the behavior of an area-preserving map around a fixed point and in the number and type of critical points and periodic orbits of an area-preserving flow, the fixed-point index of a map and the index of a singularity or a closed orbit of a flow can yield much information about the map or flow. For example, the fact that the index of an isolated singularity of an area-preserving flow can never be greater than +1 has aided in setting a lower bound for the number of stationary points of certain area-preserving flows. It has also been a useful necessary condition for a flow to be area-preserving. For these reasons, the conjecture that the fixed-point index of an area-preserving homeomorphism of a 2-manifold is always less than or equal to +1 has drawn attention. In this paper, we answer this conjecture in the affirmative for smooth maps and then put this bound to work to show that certain maps must have at least two fixed points and certain flows at least two periodic orbits. An important application is the following generalization of a famous theorem of Liapunov: a Hamiltonian vectorfield on $\mathrm{M}^{4}$ must have two distinct one-parameter families of periodic orbits around a non-degenerate minimum (or maximum) of the Hamiltonian, even when the pure imaginary characteristic exponents are in resonance.

Conversations with Jean Martinet, Ken Meyer, Charles Titus, and Alan Weinstein were very helpful in the preparation of this paper. This paper is a simplification and expansion of [15]. Many of the improvements over the presentation in [15], especially Theorem 4, are based on suggestions of K. Meyer.

\section{The Index for Flows and Transformations}

Let $\dot{x}=G(x)$ be a continuous vectorfield on a manifold $M^{n}$ with isolated singularity $x_{0}$. Since we are interested only in local behavior,

* Supported in part by the Institute for Science and Technology at the University of Michigan and by NSF Grant GP 29110. 
we can assume that $x_{0} \in U$ an open ball in $\mathbb{R}^{n}$, that $G: U \rightarrow \mathbb{R}^{n}$, and that $U \cap G^{-1}(0)=\left\{x_{0}\right\}$. The following are equivalent definitions of the index of vectorfield $G$ at $x_{0}$ :

a) The local degree of $G$ at $x_{0}$, i.e., if $U$ is a ball of radius $r$ and $S^{n-1}=\partial U$, the index is the degree of the map $\bar{G}: S^{n-1} \rightarrow S^{n-1}$ by $\bar{G}(x)=\frac{r G(x)}{|G(x)|}+x_{0}$, for $x \in \partial U$;

b) the intersection number of $G(U)$ and the zero section in the tangent bundle of $M$;

c) if $G$ is smooth, the index is $\sum_{x \in G^{-\mathrm{i}}(b)}\{$ sign $\operatorname{det} D G(x)\}$, where $b$ is a

d) in 2-dimensions, the index is $1+\frac{1}{2}[E-H]$ where $E$ is the number of elliptic regions about $x_{0}$ and $H$ is the number of hyperbolic regions, or is +1 if $x_{0}$ is a rotation point for the flow [Poincaré Index Formula].

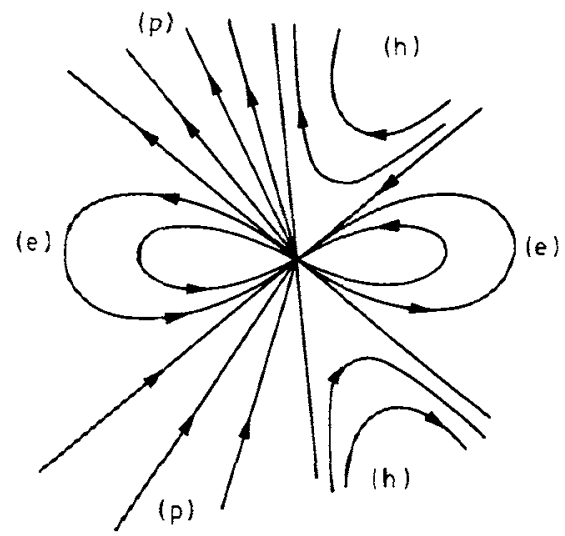

Fig. 1. Flow of $G$ about $x_{0}$ with elliptic regions $(e)$, hyperbolic regions $(h)$, and parabolic regions $(p)$

On the other hand, let $f: M \rightarrow M$ be a continuous homeomorphism with isolated fixed point $m_{0}$. The following are equivalent definitions of the index of $f$ at $m_{\mathbf{0}}$ :

e) The local degree of $1_{M}-f$ at $m_{0}$, i.e., if $m_{0}$ corresponds to the origin in ball $U$ of radius $r$, and if it is the only fixed point in $U$, then the index is the degree of the mapping $S^{n-1} \rightarrow S^{n-1}$, given by $x \mapsto r \frac{x-f(x)}{|x-f(x)|}$, where
$\partial U=S^{n-1}$;

f) the intersection number of the graph of $f$ with the diagonal at $\left(m_{0}, m_{0}\right)$ in $M \times M$; 
g) The Lefschetz number of $(f \mid V)_{\star}: H(V ; Q) \rightarrow H(f V ; Q)$, where $m_{0}$ is the only fixed point in $V$.

Among the many references for these definitions are [4-8], and [12].

\section{The Index for an Area-Preserving Flow}

Proposition 1. If $p$ is an isolated stationary point of an area-preserving flow $X$, the index of $X$ at $p$ is $\leqq+1$.

There are two quick ways of verifying this proposition. One can use definition d) above and observe that an area preserving flow cannot have any elliptic or parabolic regions about a stationary point. Alternatively, one can note that in a ball $U$ about $p$ with coordinates $(x, y)$ there is a real-valued function $H(x, y)$ such that the vectorfield $X$ is $\dot{x}=\frac{\partial H}{\partial y}, \dot{y}=-\frac{\partial H}{\partial x},($ see [1]) and then follow the argument in Hartman $[7$, p. 173]

Note that Proposition 1 holds also for gradient vector fields since the vectorfield $\dot{x}=\frac{\partial H}{\partial y}, \dot{y}=-\frac{\partial H}{\partial x}$ is perpendicular at each point to the corresponding gradient vector field $\dot{x}=\frac{\partial H}{\partial x}, \dot{y}=\frac{\partial H}{\partial y}$.

\section{Parameterizing Diffeomorphisms by Vector Fields}

Let $C^{k}\left(\mathbb{R}^{n}, \mathbb{R}^{n}\right)$ be the space of $C^{k}$ maps $\mathbb{R}^{n} \rightarrow \mathbb{R}^{n}$ with the fine $C^{k}$ topology $([11]), k \geqq 1$. Let $D^{k}\left(\mathbb{R}^{n}, \mathbb{R}^{n}\right)$ be the open subspace consisting of the $C^{k}$ diffeomorphisms, with the identity map $I \in D^{k}\left(\mathbb{R}^{n}, \mathbb{R}^{n}\right)$. Since $f \rightarrow \frac{1}{2}(I+f)$ is a continuous map of $D^{k}\left(\mathbb{R}^{n}, \mathbb{R}^{n}\right)$ into $C^{k}\left(\mathbb{R}^{n}, \mathbb{R}^{n}\right)$, there is a neighborhood $\mathscr{U}$ of $I$ in $D^{k}\left(\mathbb{R}^{n}, \mathbb{R}^{n}\right)$ such that if $f \in \mathscr{U}, \frac{1}{2}(I+f) \in D^{k}\left(\mathbb{R}^{n}, \mathbb{R}^{n}\right)$. Similarly, let $\mathscr{V}$ be a neighborhood of the zero map 0 in $C^{k}\left(\mathbb{R}^{n}, \mathbb{R}^{n}\right)$ such that if $\xi$ is in $\mathscr{F}, I+\frac{1}{2} \xi$ and $I-\frac{1}{2} \xi$ are in $D^{k}\left(\mathbb{R}^{n}, \mathbb{R}^{n}\right)$. Let $\mathscr{A}=$ $\left\{(x, f(x)) \in \mathbb{R}^{n} \times \mathbb{R}^{n} \mid f \in \mathscr{U}\right\}$, an open neighborhood of the diagonal $\boldsymbol{A}$ in $\mathbb{R}^{n} \times \mathbb{R}^{n}$.

Proposition 2. a) Consider maps $F: \mathscr{U} \rightarrow C^{k}\left(\mathbb{R}^{n}, \mathbb{R}^{n}\right)$ and $G: \mathscr{U} \rightarrow$ $D^{k}\left(\mathbb{R}^{n}, \mathbb{R}^{n}\right)$ defined by $F(f)=(I-f) \circ\left[\frac{1}{2}(I+f)\right]^{-1}$ and $G(\xi)=\left(I-\frac{1}{2} \xi\right) \circ$ $\left(I+\frac{1}{2} \xi\right)^{-1}$. Then $F: \mathscr{U} \rightarrow \mathscr{V}, G: \mathscr{V} \rightarrow \mathscr{U}$, and $F$ and $G$ are inverses to each other.

b) Consider the linear map $H: \mathbb{R}^{n} \times \mathbb{R}^{n} \rightarrow \mathbb{R}^{n} \oplus \mathbb{R}^{n}=T \mathbb{R}^{n}$ by $H(x, y)=$ $\left[\frac{1}{2} x+\frac{1}{2} y, x-y\right] . H$ takes the graph of an $f$ in $\mathscr{U}$ onto the graph of $F(f)$ in $\mathscr{W}$.

c) Suppose that $f: \mathbb{R}^{n} \rightarrow \mathbb{R}^{n}$; that $f\left(x_{0}\right)=x_{0}$; that for some neighborhoods $U$ and $V$ about $x_{0}, \frac{1}{2}(I+f): U \rightarrow V$ is a surjective diffeomorphism; and that $(x, f(x)) \in \mathscr{A}$ for all $x \in U$. Then $F(f): V \rightarrow \mathbb{R}^{n}$ is defined and $F(f)(y)=x-f(x)$, where $y=\frac{1}{2}(x+f(x))$. 
$x$ is a fixed point of $f$ if and only if $y=\frac{1}{2}(x+f(x))$ is a zero of $F(f)$; and the fixed point index of $x$ for $f$ is equal to the index of $y$ as a stationary point for the vector field $F(f)$. If $n=2$ and $k \geqq 1$ and if $f: U \rightarrow \mathbb{R}^{2}$ is areapreserving, then $F(f): V \rightarrow \mathbb{R}^{2}$ is dicergence-free.

d) Likewise, if $\xi: \mathbb{R}^{n} \rightarrow \mathbb{R}^{n}, \xi\left(y_{0}\right)=0$, and $I+\frac{1}{2} \xi: V \rightarrow W$ is a surjective diffeomorphism for neighborhoods $V$ and $W$ of $y_{0}$ with $(y, \xi(y)) \in H(\mathscr{A})$ for $y \in V$, then $G(\xi): W \rightarrow \mathbb{R}^{n}$ is defined and $G(\xi)(x)=y-\frac{1}{2} \xi(y)$ where $x=y+\frac{1}{2} \xi(y)$. $y$ a zero of $G$ of index $j$ implies that $x$ is a fixed point of $G(\xi)$ of index $j$. If $n=2$ and $k \geqq 1$ and $\xi: V \rightarrow \mathbb{R}^{2}$ is dicergence-free, then $G(\xi)$ is area-preserving.

Proof. a) To see that $G \circ F$ is the identity, let $z \in \mathbb{R}^{n}$. Then,

$$
\begin{array}{rlr}
(z,(G \circ F)(f)(z)) & =\left(z, G\left(\xi_{f}\right)(z)\right) \\
& =\left(z,\left(I-\frac{1}{2} \xi_{f}\right) \circ\left(I+\frac{1}{2} \xi_{f}\right)^{-1} z\right) & \\
& =\left(\omega+\frac{1}{2} \xi_{f}(\omega), \omega-\frac{1}{2} \xi_{f}(\omega)\right), \quad \text { where } \omega=\left(I+\frac{1}{2} \xi_{f}\right)^{-1} z, \\
& =\left(\frac{1}{2}(y+f(y))+\frac{1}{2}(y-f(y)), \frac{1}{2}(y+f(y))-\frac{1}{2}(y-f(y))\right), \\
& =(y, f(y)) . & \text { where } \omega=\frac{1}{2}(y+f(y))
\end{array}
$$

So, $z=y$ and $(G \circ F)(f)(z)=f(z)$.

Similarly, $F(G(\xi))=\xi$.

b) $\left.H(x, f(x))=\left[\frac{1}{2}(x+f x)\right), x-f(x)\right]$

$$
=\left[y,(I-f) \circ\left[\frac{1}{2}(I+f)\right]^{-1} y\right], \quad \text { where } \frac{1}{2}(x+f(x))=y .
$$

c) Clearly, $F(f)(y)=0$ if and only if $x=f(x)$, where $y=\frac{1}{2}(x+f(x))$. $H$ takes the graph of $f \mid U$ onto the graph of $F(f) \mid V . H$ also takes the graph of $I$ to the graph of 0 . Using definition b) for the index of a zero of a vector field, definition f) for the index of a fixed point of a map, and the fact that $H$ preserves intersection numbers, one finds that the index of a zero of $F(f)$ is the same as the index of the corresponding fixed point of $f$. For the last sentence of $\mathrm{c}$ ), we use the fact that a transformation $f$ of the plane is area-preserving if and only if its Jacobian, det $D f(x)$, is +1 for all $x$; and the fact that $\operatorname{div} \xi=\operatorname{trace} D \xi$. So, if $\xi=F(f)$,

$D \xi(x)=D(I-f)_{\left[\frac{1}{2}(I+f)\right]^{-1} x} \circ D\left[\frac{1}{2}(I+f)\right]^{-1}(x)$

$=D(I-f)_{y} \circ\left[D\left(\frac{1}{2}(I+f)\right)_{y}\right]^{-1}, \quad$ where $y=\left[\frac{1}{2}(I+f)\right]^{-1}(x)$

$$
=\left[\begin{array}{cc}
1-\frac{\partial f_{1}}{\partial y_{1}} & -\frac{\partial f_{1}}{\partial y_{2}} \\
-\frac{\partial f_{2}}{\partial y_{1}} & 1-\frac{\partial f_{2}}{\partial y_{2}}
\end{array}\right] \circ \frac{1}{\operatorname{det} D\left[\frac{1}{2}(I+f)\right]_{y}}\left[\begin{array}{ccc}
\frac{1}{2}\left(1+\frac{\partial f_{2}}{\partial y_{2}}\right) & -\frac{1}{2} \frac{\partial f_{1}}{\partial y_{2}} \\
-\frac{1}{2} \frac{\partial f_{2}}{\partial y_{1}} & \frac{1}{2}\left(1+\frac{\partial f_{1}}{\partial y_{1}}\right)
\end{array}\right]
$$


$=\frac{1}{\operatorname{det} D\left[\frac{1}{2}(I+f)\right]_{y}}\left[\begin{array}{lr}\frac{1}{2}\left(1-\operatorname{det} D f(y)+\frac{\partial f_{1}}{\partial y_{2}}-\frac{\partial f_{1}}{\partial y_{1}}\right) & -\frac{\partial f_{1}}{\partial y_{2}} \\ -\frac{\partial f_{2}}{\partial y_{1}} & \frac{1}{2}\left(1-\operatorname{det} D f(y)+\frac{\partial f_{1}}{\partial y_{1}}-\frac{\partial f_{2}}{\partial y_{2}}\right)\end{array}\right]$.

So, $\operatorname{div} \xi(x)=\operatorname{trace} D \xi(x)=0$ for all $x$ if and only if $\mathbf{1}=\operatorname{det} D f(y)$ for all $y$.

d) The first part of d) is proven as the first part of c) was. For the last sentence, if $f=G(\xi)$,

$$
\begin{aligned}
D f(x) & =D\left(I+\frac{1}{2} \xi\right)_{\left(I-\frac{1}{2} \xi\right)^{-1} x} \circ D\left(I-\frac{1}{2} \xi\right)^{-1} x \\
& =D\left(I+\frac{1}{2} \xi\right)_{y} \circ\left[D\left(I-\frac{1}{2} \xi\right)_{y}\right]^{-1}, \quad \text { where } y=\left(I-\frac{1}{2} \xi\right)^{-1} x .
\end{aligned}
$$

So,

$$
\operatorname{det} D f(x)=\frac{\operatorname{det} D\left(I+\frac{1}{2} \xi\right)_{y}}{\operatorname{det} D\left(I-\frac{1}{2} \xi\right)_{y}}=\frac{1+\frac{1}{2} \operatorname{div} \xi(y)+\frac{1}{4} \operatorname{det} D \xi(y)}{1-\frac{1}{2} \operatorname{div} \xi(y)+\frac{1}{4} \operatorname{det} D \xi(y)} .
$$

$\operatorname{det} D f(x)=1$ for all $x$ if and only if $\operatorname{div} \xi(y)=0$ for all $y$. Q.E.D.

Proposition 2 describes a "coordinate chart" from a neighborhood of the identity in the group of diffeomorphisms of $\mathbb{R}^{n}$ to a neighborhood of zero in the Banach space of smooth vector fields on $\mathbb{R}^{n}$. (This chart is not smooth since $F$ and $G$ involve inversion.) For $n=2$, this chart shows that the space of area-preserving diffemorphisms of the plane is a submanifold modelled on the subspace of divergence-free vectorfields. Both of these results are special cases of much more general results in symplectic geometry in the work of Weinstein $[18,21]$. A symplectic manifold $M$ is one that supports a closed non-degenerate 2 -form $\Omega$. $\Omega$ non-degenerate means that $\tilde{\Omega}: T M \rightarrow T^{*} M$ by $\left.\tilde{\Omega}(X)=X\right\lrcorner \Omega$ is an isomorphism. If $(M, \Omega)$ is a symplectic manifold, there is a natural symplectic 2-form $\Omega_{1}$ on $T M$ and a natural symplectic 2 -form $\Omega_{2}$ on $M \times M$. In [18], Weinstein describes a diffeomorphism $F$ of a neighborhood of the diagonal $\Delta$ in $\left(M \times M, \Omega_{2}\right)$ onto a neighborhood of the zero section $Z$ in $\left(T M, \Omega_{1}\right)$.F is symplectic in that $F^{*} \Omega_{1}=\Omega_{2}$. As a result, $F$ maps the graphs of symplectic diffeomorphisms $f$ of $M$ (i.e., $f: M \rightarrow M$ with $f^{*} \Omega=\Omega$ ) near the identity onto the graphs of infinitesimally symplectic vectorfields $X$ (i.e., $X: M \rightarrow T M$ such that $L_{X} \Omega=0$ ) near the zero vector field. In two dimensions, symplectic means area-preserving if one chooses $\Omega$ to be the area element.

Meyer uses such a parametrization of symplectic diffeomorphisms by symplectic (locally Hamiltonian) vector fields to study generic bifurcations of periodic points of area-preserving maps in [9] and to write canonical forms for symplectic and Hamiltonian matrices in [10]. 


\section{The Fixed Point Index of an Area-Preserving Map}

In this section, we compute the possibilities for the index of a fixed point of $C^{1}$ area-preserving map $f$ of a 2 -manifold. Since this is purely a local problem, we can suppose without loss of generality that $U$ is a convex neighborhood of the origin 0 in $\mathbb{R}^{2}$, that $f: U \rightarrow \mathbb{R}^{2}$ has 0 as its only fixed point, and that det $D f(x)=1$ for all $x$ in $U$.

Since $\operatorname{det} D f(0)$ is the product of the eigenvalues $\lambda, \mu$ of $D f(0)$, there are three possibilities for these eigenvalues: a) $0<|\lambda|<1<|\mu|$ (hyperbolic case), b) $|\lambda|=|\mu|=1$ but $\lambda \neq 1$ (elliptic case), and c) $\lambda=\mu=1$ (parabolic case).

Proposition 3. With $f: U \rightarrow \mathbb{R}^{2}$ as above, the index of 0 in case a) is \pm 1 and the index in case b) is +1 .

Proof. To compute the index, we use the fact that the index is a homotopy invariant. More precisely, if $f_{t}$ is a continuous one-parameter family of mappings of $U$ into $\mathbb{R}^{2}$ for $0 \leqq t \leqq 1$ with 0 an isolated fixed point for each $f_{t}$, the index of 0 for $f_{0}$ equals the index of 0 for $f_{1}$. (See Dold [4].)

$$
\text { Let } f_{\varepsilon}(x)=\frac{1}{\varepsilon} f(\varepsilon x) \text { for } 0<\varepsilon \leqq 1 ; f_{0}(x)=D f(0) x \text {. If } f(x)=D f(\mathbf{0}) x+R(x)
$$

is the Taylor expansion of $f$ where $R=o(|x|)$, then $f_{\varepsilon}(x)=D f(0) x+S(\varepsilon, x)$, where $S$ is $C^{1}$ in $\varepsilon$ and $x$ and $S(0, x) \equiv 0$. So, $f_{\varepsilon}$ is a homotopy of $f$ for $0 \leqq \varepsilon \leqq 1 . f_{\varepsilon}(x)=x$ for $x \in U$ if and only if $f(\varepsilon x)=\varepsilon x$ if and only if $x=0$, provided 1 is not an eigenvalue of $D f(0)$. So, in cases a) and b), the index of $f$ at 0 is the same as the index of $D f(0)$ at 0 .

In case a), $D f(0)$ moves points as in Fig. 2 with a possible flip about the origin. Here, $E_{\lambda}$ is the eigenspace of $\lambda$ and $E_{\mu}$ the eigenspace of $\mu$. Using definition e) for the fixed point index, one computes easily that the index of 0 in case a) is -1 if $D f(0) \mid E_{\mu}$ preserves the natural orientation of $E_{\mu}$ and is +1 if $D f(0) \mid E_{\mu}$ reverses this orientation. For a more general theorem, see Proposition 4.11 in Smale [17].

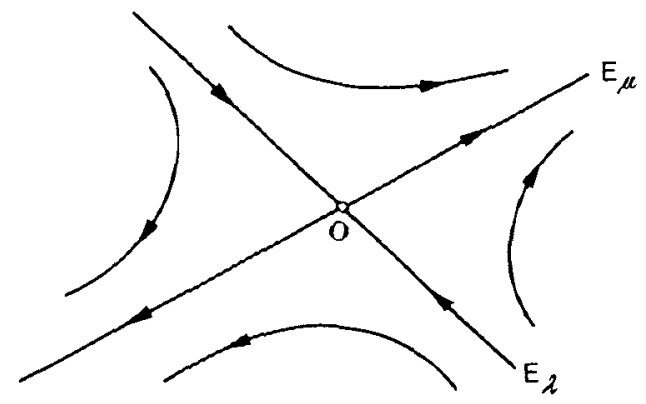

Fig. 2 
In case $b), D f(0)$ has the matrix $\left(\begin{array}{rr}\cos \theta & \sin \theta \\ -\sin \theta & \cos \theta\end{array}\right)$ for some $\theta \in(0,2 \pi)$ or is similar to $\left(\begin{array}{rr}-1 & \eta \\ 0 & -1\end{array}\right)$. Using the same techniques as in case a), one computes that the index of 0 for the rotation matrix is +1 . By letting $\eta \rightarrow 0$ in $\left(\begin{array}{rr}-1 & \eta \\ 0 & -1\end{array}\right)$ and using the invariance of index under homotopy, one finds that the index of 0 is also +1 in this situation.

We come now to the main goal of this paper.

Theorem 1. Let $f: N \rightarrow N$ be a $C^{k}$ area-preserving transformation of a 2-manifold $N, k \geqq 1$. If $p$ is an isolated fixed point of $f$, then the index of $f$ at $p$ is $\leqq+1$.

Proof. As described in the first paragraph of this section, we are led to consider $f: U \rightarrow \mathbb{R}^{2}$ where $U$ is a neighborhood of 0 in $\mathbb{R}^{2}$. By Proposition 3, we need only investigate the case where $D f(0)=\left(\begin{array}{ll}1 & \eta \\ 0 & 1\end{array}\right)$. Now, $D\left(\frac{1}{2}(I+f)\right)(0)=\frac{1}{2}(I+D f(0))=\left(\begin{array}{cc}1 & \eta / 2 \\ 0 & 1\end{array}\right)$. By the inverse function theorem, there are neighborhoods $U_{1}$ and $V$ such that $f \mid U_{1}$ is a diffeomorphism and $\frac{1}{2}(I+f) \mid U_{1}: U_{1} \rightarrow V$ is a surjective diffeomorphism, where $(x, f(x)) \in \mathscr{A}$ (of Proposition 2) for $x \in U_{1}$. Using Proposition 2, construct divergence-free vector field $F(f)$ on $V$. Since the fixed-point index of $f$ at 0 is equal to the index of 0 as a singularity of $F(f)$, this fixed-point index is $\leqq+1$ by Proposition 1 .

\section{Elementary Applications}

As discussed in the introduction, the fixed point index is an important tool in obtaining lower bounds for the number of fixed points of maps of certain manifolds $M$-mainly because of the fact that if $f: M \rightarrow M$ is a map homotopic to identity with isolated fixed points or $\xi$ is a vectorfield on $M$ with isolated singularities, then the Euler characteristic is equal to the sum of the fixed-point indices of $f$ and to the sum of the indices of the singularities of $\xi$ (see $[4,6,8,12]$ ). For example, since the Euler characteristic of the 2-sphere $S^{2}$ is +2 , every map $f: S^{2} \rightarrow S^{2}$ homotopic to identity must have a fixed point and every vector field on $S^{2}$ must have a zero. Proposition 1 and Theorem 1 yield the following strengthening of this result in the area-preserving case, since an area-preserving map has degree 1 and so by Hopf's Theorem is homotopic to identity.

Theorem 2. If $f: S^{2} \rightarrow S^{2}$ is a $C^{k}$ area-preserving map of the 2-sphere, $k \geqq 1$, then $f$ has at least 2 fixed points. If $\xi$ is a gradient, Hamiltonian, or divergence-free vector field on $S^{2}$, then $\xi$ has at least two singularities. 
If one could prove the analogue of Theorem 2 for $\mathbf{C}^{0}$ maps, then one could show that an area preserving map $g$ of the open two disk onto itself must have a fixed point, without using the techniques of Brouwer for mappings of the plane. One would construct from $g$ an area-preserving map $\hat{g}$ of $S^{2}$ with a fixed point at infinity and use the $C^{0}$ analogue of Theorem 2 to point out that $\hat{g}$ must have another fixed-point.

As another simple application of Theorem 1, we obtain the following clarification of Poincarés Geometric Theorem.

Theorem 3. Let $T$ be a $C^{1}$ area-preserving transformation of the annulus that leaves the boundary circles $C_{1}$ and $C_{2}$ invariant with $T \mid C_{i}$ a simple rotation of the circle. If $T$ has a fixed point of index $-n$, then $T$ has at least $n+1$ fixed points.

(Birkhoff [3] has shown that if $T$ rotates the boundary circles in opposite directions, then $T$ must have at least one fixed point.)

Proof. The sum of the indices throughout the annulus is zero. To see this, let $D_{1}$ and $D_{2}$ be the 2-disks bounded by the simple closed curves $C_{1}$ and $C_{2}$ respectively, with $D_{1} \subset D_{2}$. Construct $g: D_{1} \rightarrow D_{1}$ with a single fixed point such that $g\left|C_{1}=T\right| C_{1}$, thus extending $T$ to a map $\hat{T}: D_{2} \rightarrow D_{2}$. Using definition e) of the index as in part a) of Proposition 2, one notes that the sum of the fixed-point indices of $g: D_{1} \rightarrow D_{1}$ is +1 , as is the corresponding sum for $\hat{T}: D_{2} \rightarrow D_{2}$. Therefore, the sum of the fixed-point indices in $\overline{D_{2}}-\overline{D_{1}}$, the annulus, is zero. But by Theorem 1 , each index in the annulus is $\leqq+1$.

\section{Families of Periodic Orbits for Hamiltonian Systems}

Our main tool in this section will be the index for periodic orbits of a smooth vector-field $X$ as described and utilized by Fuller [5]. Let $M$ be a smooth manifold with $\phi: M \times(-\infty, \infty) \rightarrow M$ the flow of $X$ on $M$. Let $\Omega$ be an open set in $M \times(0, \infty)$ such that

a) $\bar{\Omega}$ is compact in $M \times(0, \infty)$;

b) if $(x, t) \in \bar{\Omega} \backslash \Omega$, then $\phi(x, t) \neq x$;

c) if $(x, t) \in \bar{\Omega}, X(x) \neq 0$.

For such $\Omega$, Fuller defines a rational number $i(\Omega)$, the index of $\Omega$, with the following properties:

1) if $\Omega=\Omega_{1} \cup \Omega_{2}, \Omega_{1} \cap \Omega_{2}=\phi$, and $\Omega_{1}$ and $\Omega_{2}$ satisfy a), b), c), then $i(\Omega)=i\left(\Omega_{1}\right)+i\left(\Omega_{2}\right)$,

2) if $X_{0}$ and $\Omega_{0}$ are continuously deformed to $X_{\alpha}$ and $\Omega_{\alpha}$ such that each $\Omega_{\alpha}$ satisfies a), b), c) for $X_{\alpha}, \alpha \in[0,1]$, then $i\left(\Omega_{\alpha}\right)$ is independent of $\alpha$. 
3) Suppose $\Omega=U \times\left(k t_{0}-\frac{t_{0}}{2}, k t_{0}+\frac{t_{0}}{2}\right)$ where $k$ is an integer, $U$ is an open set in $M$ that contains only one periodic orbit $\gamma$; and $\gamma$ has least period $t_{0}$. Let $D$ be an $(n-1)$-disk meeting $\gamma$ transversally at $x_{0}$, with $D \cap \gamma=\left\{x_{0}\right\}$. Let $T$, mapping a neighborhood of $x_{0}$ in $D$ into $D$, be the Poincare first-return map for $X$. Then, $i(\Omega)$ equals $1 / k \times$ (fixedpoint index of $T^{k}$ at $x_{0}$ ).

4) If $\Omega=U \times\left(t_{1}, t_{2}\right)$ and $i(\Omega) \neq 0$, then $U$ contains a periodic orbit of period $\tau \in\left(t_{1}, t_{2}\right)$.

Fuller applies his index in [5] to prove the following theorem of Seifert.

Proposition 4 (see Seifert [13]). Let $X$ be a vector field on $S^{3}$ with every orbit a non-trivial periodic orbit of least period $2 \pi$ and with the orbit space homeomorphic to $S^{2}$. Let $\Omega=S^{3} \times(\pi, 3 \pi)$. Then $i(\Omega)=+2$. Therefore, perturbations of $X$ have at least one periodic orbit.

Fuller's proof involves i) constructing a vectorfield $G$ on $S^{2}$ where flow fixes the north and south poles and sends the other points down the meridians, ii) lifting $G$ to $\hat{G}$ on $S^{3}$, using the fact that $S^{2}$ is the orbit space of $X$, iii) noting that $X_{\varepsilon}=X+\varepsilon \hat{G}$ has only two periodic orbits, both of index one, for $\varepsilon \neq 0$, small, iv) using the invariance of $i(\Omega)$ under homotopy.

Let us now apply Fuller's index to the search for families of periodic orbits of Hamiltonian dynamical systems. Let $H: M^{4} \rightarrow \mathbb{R}$ be a smooth (Hamiltonian or energy) function on a four-dimensional phase space, with $p \in M^{4}$ an isolated critical point. Choose canonical coordinates about $p$ so that we can view $H$ as defined on a neighborhood of $\mathbf{0}$ in $\mathbb{R}^{4}$ with $H(\mathbf{0})=0$ and $\operatorname{grad} H(\mathbf{0})=0$. Let $J$ be the matrix

$$
\left(\begin{array}{rrrr}
0 & 0 & 1 & 0 \\
0 & 0 & 0 & 1 \\
-1 & 0 & 0 & 0 \\
0 & -1 & 0 & 0
\end{array}\right) .
$$

Then, the corresponding Hamiltonian equations for a system with energy function $H$ is $\dot{\mathbf{x}}=J \circ \operatorname{grad} H(\mathbf{x}) \equiv X_{H}(\mathbf{x})$. Suppose the characteristic multipliers of $X_{H}$ at $\mathbf{0}$, i.e., the eigenvalues of the linear map $D X_{H}(\mathbf{0})$, are pure imaginary: $\pm i \omega_{1}$ and $\pm i \omega_{2}$, where $\omega_{1}$ and $\omega_{2}$ are real and $\left|\omega_{1}\right|<\left|\omega_{2}\right|$. In this case, by a standard diagonalization technique $[14, \S 15]$, there are canonical coordinates $\left(x_{1}, x_{2}, y_{1}, y_{2}\right)$ in $U$ such that

$$
H\left(x_{1}, x_{2}, y_{1}, y_{2}\right)=\frac{\omega_{1}}{2}\left(x_{1}^{2}+y_{1}^{2}\right)+\frac{\omega_{2}}{2}\left(x_{2}^{2}+y_{2}^{2}\right)+R(\mathbf{x}, \mathbf{y})
$$


where $R$ is $0\left(|(\mathbf{x}, \mathbf{y})|^{2}\right)$. The linearized equations, $\dot{\mathbf{x}}=D X_{\boldsymbol{H}}(\mathbf{0}) \mathbf{x}$, are the Hamiltonian equations for the Hamiltonian

$$
H_{0}\left(x_{1}, x_{2}, y_{1}, y_{2}\right)=\frac{\omega_{1}}{2}\left(x_{1}^{2}+y_{1}^{2}\right)+\frac{\omega_{2}}{2}\left(x_{2}^{2}+y_{2}^{2}\right),
$$

the quadratic part of $H$. These linear equations are:

$$
\dot{x}_{1}=\omega_{1} x_{1}, \quad \dot{x}_{2}=\omega_{2} y_{2}, \quad \dot{y}_{1}=-\omega_{1} x_{1}, \quad \dot{y}_{2}=-\omega_{2} x_{2} .
$$

If we choose coordinates $\left(z_{1}, z_{2}\right) \in \mathbb{C} \times \mathbb{C}$ in $U$ where $z_{k}=x_{k}+i y_{k}, k=1,2$, then $\dot{\mathbf{x}}=D X_{H}(\mathbf{0}) x=X_{H_{0}}(\mathbf{x})$ becomes $\dot{z}_{k}=i \omega_{k} z_{k}$ with solutions

$$
z_{1}(t)=z_{1} e^{-i \omega_{1} t}, \quad z_{2}(t)=z_{2} e^{-i \omega_{2} t} .
$$

Here, the (generalized) eigenspace $z_{2}=0$ for $\pm i \omega_{1}$ is a one-parameter family of periodic orbits of $\dot{z}=D X_{H}(\mathbf{0}) \mathbf{z}$ with period $\left|\frac{2 \pi}{\omega_{1}}\right|$ and the (generalized) eigenspace $z_{1}=0$ of $\pm i \omega_{2}$ is a one-parameter family of periodic orbits of period $\left|\frac{2 \pi}{\omega_{2}}\right|:$ A famous theorem of Liapunov (see [14]) asserts that there still exists a smooth one-parameter family of orbits for the non-linear equation $\dot{\mathbf{x}}=X_{H}(\mathbf{x})$ near $z_{1}=0$ with period near $\left|\frac{2 \pi}{\omega_{2}}\right|$; and if $\frac{\omega_{2}}{\omega_{1}}$ is not an integer, there is another such family near $z_{2}=0$ with period $\left|\frac{2 \pi}{\omega_{1}}\right|$.

In Theorem 4 below, we use Theorem 1 and Fuller's index to show the existence of this second family even if $\frac{\omega_{2}}{\omega_{1}}$ is an integer (even 1), provided that the Hessian of $H$ at $\mathbf{0}$ is positive (or negative) definite. Weinstein $[19,20]$ has used much more complex techniques to obtain the definitive results in this situation. Berger [2] has used techniques of the calculus of variations to exhibit families of periodic orbits in similar situations. On the other hand, for each integer $n \geqq 1$, there exists $H: \mathbb{R}^{4} \rightarrow \mathbb{R}$ with $H(0)=0, D H(0)=0, D^{2} H(0)$ indefinite, $\frac{\omega_{2}}{\omega_{1}}=-n$, and $X_{H}$ having
only one family of periodic orbits [16].

Theorem 4. Let $H: M^{4} \rightarrow \mathbb{R}$ be a smooth $\left(C^{2}\right)$ Hamiltonian function on a 4-dimensional phase space with $p \in M$ a non-degenerate minimum (or maximum) of $H$ and $H(p)=c_{0}$. Then, for $c$ near $c_{0}$, the Hamiltonian system $\dot{\mathbf{x}}=\boldsymbol{J} \circ \operatorname{grad} \boldsymbol{H}(\mathbf{x})$ has at least two periodic orbits on each energy surface $H^{-1}(c)$. 
Proof. As above, choose canonical coordinates $\left(z_{1}, z_{2}\right)$ in $\mathbb{C} \times \mathbb{C}$ about $\mathbf{p}$ so that $\mathbf{p}$ corresponds to 0 and in this neighborhood

$$
H\left(z_{1}, z_{2}\right)=\frac{\omega_{1}}{2} z_{1} \bar{z}_{1}+\frac{\omega_{2}}{2} z_{2} \bar{z}_{2}+R\left(z_{1}, z_{2}\right), \quad\left|\omega_{1}\right| \leqq\left|\omega_{2}\right|,
$$

as in (*). Since $\mathbf{p}$ is a non-degenerate minimum (maximum), $D^{2} H(0)$ is positive (negative) definite and $\omega_{1}$ and $\omega_{2}$ are both positive (negative). Without loss of generality, we assume $\mathbf{p}$ is a minimum. (Otherwise, work with $-H$.) Consider the one-parameter family of Hamiltonians

$$
\begin{aligned}
& H_{\varepsilon}(\mathbf{z})=\frac{1}{\varepsilon^{2}} H(\varepsilon \mathbf{z}) \quad \text { for } 0<\varepsilon \leqq 1, \\
& H_{0}(\mathbf{z})=\frac{\omega_{1}}{2} z_{1} \bar{z}_{1}+\frac{\omega_{2}}{2} z_{2} \bar{z}_{z} \quad \text { for } \varepsilon=0 .
\end{aligned}
$$

Since $R\left(z_{1}, z_{2}\right)$ is $0\left(|\mathbf{z}|^{2}\right), H_{\varepsilon}(z)$ is smooth in $\varepsilon$. Let $X_{H_{\varepsilon}}$ be the Hamiltonian vector field $\dot{z}=J \circ \operatorname{grad} H_{\varepsilon}(\mathbf{z})$. For $\varepsilon \neq 0$, multiplication by $\varepsilon$ takes integral curves of $X_{H_{\varepsilon}}$ on $H_{\varepsilon}^{-1}(1)$ onto integral curves of $X_{H} \equiv X_{H_{1}}$ on $H^{-1}\left(\varepsilon^{2}\right)$. So a periodic orbit for $X_{H_{\varepsilon}}$ on $H_{\varepsilon}^{-1}(1)$ corresponds to periodic orbit of $X_{H}$ on $H^{-1}\left(\varepsilon^{2}\right)$. [We are using the fact that $H_{\varepsilon}$ is constant on integral curves of $X_{\boldsymbol{H}_{\varepsilon}}$.]

Consequently, using the homotopy $H_{\varepsilon}$ allows us to view $X_{\boldsymbol{H}}$ as a perturbation of the linear system $X_{H_{0}}(\mathbf{z})=D X_{H}(0)(\mathbf{z}) . X_{H_{0}}$ has solution $\left(z_{10} e^{-i \omega_{1} t}, z_{20} e^{-i \omega_{2} t}\right)$. If $\frac{\omega_{2}}{\omega_{1}}$ is not an integer, Liapunov's Theorem asserts that $X_{H}$ has 2 families of periodic orbits, parametrized by energy. [In this case, on $H_{0}^{-1}(c)$ the periodic orbits

$$
\left\{\left(0,\left|\frac{2 c}{\omega_{2}}\right|^{\frac{\pi}{2}} e^{-i \omega_{2} t}\right) \mid t \in \mathbb{R}\right\} \text { and }\left\{\left(\left|\frac{2 c}{\omega_{1}}\right|^{\frac{1}{2}} e^{-i \omega_{1} t}, 0\right) \mid t \in \mathbb{R}\right\}
$$

have non-zero Fuller index and so are preserved in perturbing $X_{H_{0}}$ to $\left.X_{H_{\varepsilon}} \cdot\right]$

We treat the case where $\frac{\omega_{2}}{\omega_{1}}$ is an integer. Since $X_{\tilde{H}}$ has the same phase portrait as $X_{H}$ where $\tilde{H}=\frac{1}{\omega_{1}} H$, we can suppose that $\omega_{1}=1$ and $\left.\omega_{2}=\omega \in \mathbb{N} . H_{0}^{-1}\left(\frac{1}{2}\right)=\left\{\left(z_{1}, z_{2}\right) \in \mathbb{C} \times \mathbb{C}|| z_{1}\right\}^{2}+\omega\left|z_{2}\right|^{2}=1\right\}$ is diffeomorphic to the 3 -sphere $S^{3}$. Each orbit $\left(z_{10} \mathrm{e}^{-i t}, z_{20} e^{-i \omega t}\right)$ has least period $2 \pi$, except the orbit $\gamma_{0}=\left\{\left(0, \sqrt{\frac{1}{\omega}} e^{-i \omega t}\right) \mid t \in \mathbb{R}\right\}$ which has least period $\frac{2 \pi}{\omega}$. Let $T_{a}$ be the torus $\left\{\left(z_{1}, z_{2}\right) \in H_{0}^{-1}\left(\frac{1}{2}\right)|| z_{1} \mid=a^{\frac{1}{2}}\right.$ (and thus $\left.\left.\left|z_{2}\right|^{2}=\frac{1-a}{\omega}\right)\right\}$, $0<a<1 . H_{0}^{-1}\left(\frac{1}{2}\right)$ is the disjoint union of the $T_{a}^{\prime}$ 's for $0<a<1$ and the orbits 
$\gamma_{0}$ and $\gamma_{1}$, where $\left.\gamma_{1}=\left\{e^{-i t}, 0\right) \mid t \in \mathbb{R}\right\}$. Each $T_{a}$ is invariant under the flow of $X_{H_{0}}$ on $H_{0}^{-1}\left(\frac{1}{2}\right)$.

We want to compute $i(\Omega)$ where $\Omega=H_{0}^{-1}\left(\frac{1}{2}\right) \times\left(2 \pi-\frac{\pi}{\omega}, 2 \pi+\frac{\pi}{\omega}\right)$.

For $\omega=1$ (characteristic exponents of $X_{H}$ equal in pairs), $i(\Omega)=+2$ by the calculation of Proposition 4. So in this case, using 2) of the properties of $i(\Omega), i\left(\Omega_{\varepsilon}\right)=+2$ where $\Omega_{\varepsilon}=H_{\varepsilon}^{-1}\left(\frac{1}{2}\right) \times(\pi, 3 \pi)$; and $X_{H_{\varepsilon}}$ must have a periodic orbit of period near $2 \pi$ for $\varepsilon$ small. Using the fact that the Poincaré first-return map on an energy surface of a Hamiltonian system on $M^{4}$ is area-preserving $[1,14]$, property 3 ) of the Fuller index, and Theorem 1 , we see that the index of this orbit is at most +1 . Since $i\left(\Omega_{\varepsilon}\right)=+2$, there must be another periodic orbit on $H_{\varepsilon}^{-1}\left(\frac{1}{2}\right)$.

To compute $i(\Omega)$ when $\omega>1$, consider the smooth map $g: H_{0}^{-1}\left(\frac{1}{2}\right) \rightarrow \mathbb{R}$ by $g\left(z_{1}, z_{2}\right)=\left|z_{1}\right|^{2}$. Let $Y$ be the gradient vector field of $g$ on $H_{0}^{-1}\left(\frac{1}{2}\right)$. The circles $\gamma_{0}$ and $\gamma_{1}$ are circles of critical points of $Y$ and the flow of $Y$ takes torus $T_{a}$ to torus $T_{a^{\prime}}$ where $0<a<a^{\prime}<1$. Now, consider vector field $F_{\varepsilon}=X_{H_{0}}+\varepsilon Y$ on $S^{3}$. For $\varepsilon \neq 0$, the orbits $\gamma_{0}$ and $\gamma_{1}$ are the only periodic orbits of $F_{\varepsilon}$ and the flow of $F_{\varepsilon}$ sends points from $\gamma_{0}$ toward $\gamma_{1}$ (i.e., $\gamma_{0}$ is a source and $\gamma_{1}$ a sink). So, $i(\Omega)$ satisfies a), b), c) for each $F_{\varepsilon}$. By property 2) of $i, i(\Omega)$ for $X_{H_{0}}$ equals $i(\Omega)$ for $F_{\varepsilon}$. Let $U_{1}=\left\{\left(z_{1}, z_{2}\right) \in H_{0}^{-1}\left(\frac{1}{2}\right)|| z_{1} \mid<\frac{1}{2}\right\}$ and $U_{2}=$ the interior of its complement in $H_{0}^{-1}\left(\frac{1}{2}\right)$. For vector field $F_{\varepsilon}$,

$$
i(\Omega)=i\left(U_{1} \times\left(2 \pi-\frac{\pi}{\omega}, 2 \pi+\frac{\pi}{\omega}\right)\right)+i\left(U_{2} \times\left(2 \pi-\frac{\pi}{\omega}, 2 \pi+\frac{\pi}{\omega}\right)\right)
$$

by properties 1$)$ and 4$)$ of $i(\Omega)$

$$
=+1+\frac{1}{\omega}
$$

by property 3 ) and the fact that the fixed point index of a contraction or its inverse is +1 . Finally, for $\varepsilon>0$ and small, $H_{\varepsilon}^{-1}\left(\frac{1}{2}\right)$ is a 3 -sphere and $\Omega_{\varepsilon}=H_{\varepsilon}^{-1}\left(\frac{1}{2}\right) \times\left(2 \pi-\frac{\pi}{\omega}, 2 \pi+\frac{\pi}{\omega}\right)$ satisfies a), b), c) for $X_{H_{\varepsilon}}$. Therefore, $i\left(\Omega_{\varepsilon}\right)>1$. Again using the fact that Poincaré map for $X_{H_{\varepsilon}}$ on $H_{\varepsilon}^{-1}\left(\frac{1}{2}\right)$ is area-preserving, property 3) of $i$, and Theorem 1, we see that there must be at least two periodic orbits on $H_{\varepsilon}^{-1}\left(\frac{1}{2}\right)$. These correspond to periodic orbits of $X_{H}$ on $H^{-1}\left(\frac{\varepsilon^{2}}{2}\right)$.

As Alan Weinstein and Jean Martinet have pointed out to me, one can construct an area-preserving Poincaré map about a periodic orbit of a volume-preserving vector field $X$ on a manifold $M$. If $\eta$ is the volume form on $M$ and $L_{X} \eta=0$, one puts the volume form $\left.X\right\lrcorner \eta$ on a codimension one transverse disk. Therefore, the above techniques and Theorem 1 yield the following strengthening of Proposition 4. 
Theorem 5. Let $F$ be a vectorfield on $S^{3}$ equivalent to the vectorfield $z_{1}=-i z_{1}, z_{2}=-i k z_{2}$ on the 3-sphere $\left\{\left(z_{1}, z_{2}\right) \in \mathbb{C} \times\left.\mathbb{C}|| z_{1}\right|^{2}+k\left|z_{2}\right|^{2}=\frac{1}{2}\right\}$, where $k$ is a positive integer. Then, divergence-free perturbations of $F$ have at least two distinct periodic orbits.

\section{No Bounds in Higher Dimensions}

The following computations, worked out with Charles Titus, show that there are no restrictions on the index for volume preserving or even symplectic transformations and flows in higher dimensions. Once again, put coordinates $\left(z_{1}, z_{2}\right) \in \mathbb{C} \times \mathbb{C}$ on $\mathbb{R}^{4}$. Consider the Hamiltonian function

$$
H\left(z_{1}, z_{2}\right)=-\frac{1}{2}\left(z_{1}^{n}+\bar{z}_{1}^{n}+z_{2}^{m}+\bar{z}_{2}^{m}\right),
$$

where $n$ and $m$ are integers greater than 1. The corresponding Hamiltonian vector-field is

$$
\begin{aligned}
& \dot{z}_{1}=-2 i \frac{\partial H}{\partial \bar{z}_{1}}=i n \bar{z}_{1}^{n-1} \\
& \dot{z}_{2}=-2 i \frac{\partial H}{\partial \bar{z}_{2}}=i m \bar{z}_{2}^{m-1},
\end{aligned}
$$

or $\left(\dot{z}_{1}, \dot{z}_{2}\right)=G\left(z_{1}, z_{2}\right)$. A simple computation shows that the determinant of the Jacobian of $G$ will have the same sign in a neighborhood of 0 for each $m, n$. By definition c) of the index of a singularity of a vector field, the index of $\mathbf{0}$ for $\dot{\mathbf{z}}=G(\mathbf{z})$ is equal to the cardinality of $G^{-1}(c)$ for regular values $c$ near 0 , i.e., to $(m-1)(n-1)$. The time-one map for the flow of this vector field will be volume-preserving on $\mathbb{R}^{4}$ and have $\mathbf{0}$ as an isolated fixed point of index $(m-1)(n-1)$.

\section{References}

1. Abraham, R., Marsden, J.: Foundations of Mechanics. New York: W.A. Benjamin 1967

2. Berger, M.S.: Autonomous Perturbations of some Hamiltonian Systems, all of whose solutions are periodic. Ordinary Differential Equations - 1971 NRL-MRC Conference pp. 351-357. New York: Academic Press 1972

3. Birkhoff, G. D.: Dynamical Systems. Amer. Math. Soc. Colloq. Publ., Vol. 9, Amer. Math. Soc., Providence, R.I., 1927

4. Dold, A.: Lectures on Algebraic Topology. Berlin-Heidelberg-New York: Springer 1972

5. Fuller, F. B.: An index of fixed point type for periodic orbits. Amer. Journ. Math. 89, 133-148 (1967)

6. Greub, W., Halperin, S., Vanstone, R.: Connections, Curvature, and Cohomology. New York: Academic Press 1972

7. Hartman, P.: Ordinary Differential Equations. New York: John Wiley 1964

8. Lefschetz, S.: Algebraic Topology. Amer. Math. Soc. Colloq. Publ., Vol. 27, Amer. Math. Soc. Providence, R.I., 1942 
9. Meyer, K.: Generic bifurcation of periodic points. Trans. Amer. Math. Soc. 149, 95-107 (1970)

10. Meyer, K., Laub, A.: Canonical forms for symplectic and hamiltonian matrices, preprint.

11. Munkres, J.: Elementary Differential Topology. Ann. of Math. Studies 54, Princeton Univ. Press, Princeton, 1963

12. Pugh, C.: A generalized Poincaré Index Formula. Topology, 217-226 (1968)

13. Seifert, $\mathrm{H}$.: Closed integral curves in 3-space and isotopic two-dimensional deformations. Proc. Amer. Math. Soc. 1, 287-302 (1950)

14. Siegel, C.L., Moser, J. K.: Lectures on Celestical Mechanics. New York: Springer 1971

15. Simon, C. P.: The fixed point index of an area-preserving map is $\leq+1$, mimeographed notes, Univ. of Michigan

16. Simon, C. P., Weinstein, A.: Long period orbits and the hamiltonian averaging method, to appear

17. Smale, S.: Differentiable dynamical systems. Bull. Amer. Math. Soc. 73, 747-817(1967)

18. Weinstein, A.: Symplectic manifolds and their lagrangian sub manifolds. Advances in Math. 6, 329-346 (1971)

19. Weinstein, A.: Lagrangian submanifolds and hamiltonian systems. Annals. of Math. 98, 377-410 (1973)

20. Weinstein, A.: Normal modes for non-linear hamiltonian systems. Inventiones math. 20, 47-57 (1973)

21. Weinstein, A.: The Invariance of Poincaré's generating function for canonical transformations. Inventiones math. 16, 202-213 (1972)

\author{
Carl P. Simon \\ Department of Mathematics \\ University of Michigan \\ Ann Arbor, Michigan 48104, USA
}

(Received January 29,1974) 